\section{Características clínicas, esquemas de tratamiento, grado de control y variables asociadas a este en una cohorte de adultos con diabetes mellitus tipo 1 en un centro terciario}

\author{
BRUNO GRASSI ${ }^{1}$, LUCIANO TAPIA ${ }^{\mathrm{a}}$, ISMAEL ARACENA ${ }^{\mathrm{a}}$, \\ ROCÍO ASTUDILLO ${ }^{a}$, FERNANDA KARAa, MARGOT ALISTE ${ }^{1, b}$, \\ MARÍA TERESA ONETTO ${ }^{1, \mathrm{c}}$, PAMELA MATAMALA ${ }^{1}$
}

\section{Clinical features and management of 205 adults with type 1 diabetes mellitus}

Background: Few studies have evaluated the details of insulin therapy for type 1 diabetes mellitus (T1D) in Chile. Aim: To describe clinical features and treatment details of adults with T1D and its association with metabolic control. Material and Methods: Review of medical records of patients with T1D treated in a clinical network. Demographic and clinical features, types and doses of insulin and glycated hemoglobin levels were registered. The use flash glucose monitors (FGM) and insulin pumps (CSII) were also recorded. Results: 205 records were reviewed, with T1d lasting $12 \pm 10$ years. Twenty six percent had hypothyroidism, $1 \%$ had celiac disease, 12\% had hypertension, 20\% had dyslipidemia; $13 \%$ had diabetic retinopathy, $2 \%$ had diabetic nephropathy, $8 \%$ had neuropathy and $2 \%$ cardiovascular diseases. Mean body mass index was $25 \mathrm{~kg} /$ $\mathrm{m}^{2}$ and mean glycated hemoglobin was 8\%. Eighty-two percent used multiple daily injections, $18 \%$ used CSII and 24\% used FGM. As basal insulin, 35\% used slow acting analogs and 65\% used ultra-slow analogs. As rapid acting insulin, 69 patients used Lispro, 79 Aspart and 50 Glulisin. Bolus doses were calculated using only capillary glucose in $22 \%$, while $78 \%$ also considered carbohydrate consumption. Variables significantly associated to better control were the use of carbohydrates for dosing rapid insulin (A1c 7,85\% vs 8,59\%, $p=0,008$ ), use of CSII (A1c 7,36\% vs 8,16\%, $p=0,008)$, and basal dose $<0,4 \mathrm{U} / \mathrm{kg}(\mathrm{Alc} 7,81 \%$ vs $8,58 \%, p=0,003)$. There were no differences regarding insulin type or use of FGM. Conclusions: The use of formulas considering carbohydrates for dosing rapid insulin, use of infusion pumps and physiological doses of basal insulin are significantly associated with a better metabolic control in adults with T1d.

(Rev Med Chile 2019; 147: 451-457)

Key words: Diabetes Mellitus; Insulin Infusion Systems; Patient Education as Topic.

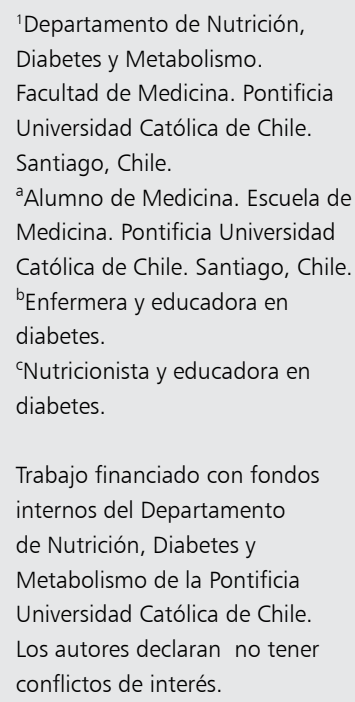

Recibido el 22 de agosto de 2018, aceptado el 3 de abril de 2019.

Correspondencia a:

Bruno Grassi Corrales

Diagonal Paraguay 362, $4^{\circ}$ Piso. Departamento de Nutrición, Diabetes y Metabolismo. Pontificia Universidad Católica de Chile.

bagrassi@gmail.com

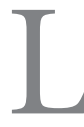

a diabetes mellitus tipo 1 (DM1) es una condición caracterizada por la destrucción $\checkmark$ de origen autoinmune de las células beta, la cual lleva a una deficiencia severa en la secreción de insulina. Esta característica fundamental en la fisiopatología de la enfermedad obliga al paciente con DM1 a remplazar la secreción de esta hormona mediante la inyección de insulina exógena ${ }^{1,2}$. 
El estudio Diabetes Control and Complications Trial (DCCT), de más de dos décadas de antigüedad, mostró por primera vez que la insulinoterapia intensiva se asocia a un mejor nivel de hemoglobina glicosilada (HbAlc) y que sus valores menores a 7\% logran reducir la aparición y progresión de complicaciones microvasculares ${ }^{3}$. Seguimientos a largo plazo de la cohorte de dicho estudio ha mostrado, además, que el control metabólico intensivo precoz se asocia a largo plazo a disminución de la morbilidad cardiovascular y a una mortalidad similar a la población general ${ }^{4}$. Sin embargo, lograr esta meta es difícil y requiere amplios esfuerzos, tanto del paciente como del equipo de salud. La publicación T1D Exchange Clinic Registry, en su última actualización de 2015, incluyó más de 16.000 pacientes, de los cuales solo 15 y $29 \%$ presentaba HbAlc menores a 7 y $7,5 \%$, respectivamente ${ }^{5}$. Lograr estos valores supone una dosificación adecuada de insulina, tanto lenta como rápida, lo que, a su vez, necesita una gran educación que permita interpretar la medición de glucosa en sangre en cada situación inmediata y frente a cada ingesta de macronutrientes. Algunas intervenciones que han mostrado mejorar el control metabólico son el uso de esquemas variables, es decir, según conteo estructurado de carbohidratos guiado por un educador especializado, y el uso de la tecnología, particularmente los microinfusores de insulina $y$ los sensores de glucosa integrados ${ }^{5-8}$. Otras intervenciones, como el uso de análogos ultralentos como insulina degludec o insulina glargina $300 \mathrm{U}$ y el uso de monitoreo flash, han mostrado mantención del control metabólico con menor riesgo de hipoglucemia ${ }^{9-11}$.

La gran cantidad de variables involucradas en el tratamiento de la DM1 genera una importante complejidad de su manejo para el paciente y condiciona, además, una dificultad en su seguimiento, en la estandarización de protocolos de manejo y en la evaluación de resultados a largo plazo por parte de los equipos de salud. Si bien existen algunos datos chilenos sobre incidencia ${ }^{12}$, sobre formas de presentación en pediatría ${ }^{13}$, comorbilidades autoinmunes ${ }^{14}$, algunas referidas a nivel de control en población pediátrica ${ }^{15} \mathrm{y}$ características del tratamiento en esta población ${ }^{16}$, en población adulta a nivel nacional se desconoce el nivel de control según edad, el tipo de esquema de tratamiento utilizado (tanto las insulinas usadas como el método de cálculo de dosis), el uso de tecnología para asistir a este tratamiento y la asociación de todas estas variables al nivel de HbAlc.

El objetivo del presente estudio es describir algunas características clínicas relevantes de un grupo en una cohorte de adultos con DM1 controlados en nuestra red de salud. Asimismo, detallar los esquemas de tratamiento e insulinas usadas, el uso de tecnología, particularmente microinfusores de insulina y monitoreo flash, y la relación con el nivel de control, medido como hemoglobina glicosilada, y la magnitud de asociación de estas variables con el control metabólico.

\section{Material y Método}

El presente estudio consiste en un estudio descriptivo retrospectivo. Fue presentado en el comité de ética y seguridad de nuestra institución, y se solicitó dispensa de consentimiento informado, dado la metodología del estudio y los datos a analizar.

Se identificaron pacientes portadores de diabetes mellitus tipo 1 del registro de casos tratados por nuestro equipo de especialistas. Las fichas electrónicas de dichos pacientes fueron revisadas en forma retrospectiva con una planilla estandarizada de análisis y registro. Se incluyeron todos aquellos pacientes que tuvieran diagnóstico consignado de DM1 o de diabetes latente autoinmune del adulto (LADA), ya sea por impresión clínica, debut con cetoacidosis diabética (CAD) o serología de DM1 presente (anticuerpos antiislote [ICA], anticuerpos antiinsulina [IAA], anticuerpos antidecarboxilasa de ácido glutámico [GAD] o anticuerpos antitirosínfosfatasa [IA2]), y que hubieran tenido al menos un control con un especialista de nuestro equipo en la Red Salud UC en el período descrito.

Se registraron para cada paciente las siguientes variables personales y antecedentes de la diabetes mellitus: fecha de nacimiento, año de debut de la DM1, hemoglobina glicosilada más reciente, tiempo de evolución, peso, talla, índice de masa corporal (IMC), presencia de comorbilidades autoinmunes (hipotiroidismo, enfermedad celíaca, insuficiencia suprarrenal u otras), hipertensión arterial y dislipidemia (según criterios vigentes), presencia de complicaciones microvasculares (neuropatía, retinopatía y nefropatía), macrovasculares y antecedente de familiares de primer grado con DM1. 
Sobre el esquema de tratamiento, se registraron las siguientes variables: dosis diaria total de insulina, dosis y tipo de insulina basal, tipo de insulina rápida, forma de dosificación de insulina rápida (dosis fija, según tabla fija de glicemias o con esquema variable según carbohidratos y glicemia), uso de monitor de glucosa flash (MGF) y uso de microinfusor de insulina de cualquier tipo (sencillo o integrado a monitoreo contínuo de glucosa). Por la metodología usada, la dosis total de insulina rápida diaria no pudo registrarse sistemáticamente.

Se testeó normalidad de la muestra, y una vez confirmada esta distribución, se procedió a realizar las comparaciones estadísticas entre medias utilizando t de Student para variables dicotómicas y test ANOVA para comparaciones múltiples. Las variables a comparar con el nivel de control metabólico, definido como $\mathrm{HbAlc}$, fueron definidas a priori: tipo de insulina basal y rápida usadas, dosis de insulina basal, forma de dosificación de insulina rápida, uso de MGF y uso de microinfusor de insulina.

\section{Resultados}

Se revisaron 283 fichas en que aparecía el diagnóstico "diabetes mellitus tipo 1" en el sistema computacional de nuestra red. Luego del análisis y tamizaje inicial, se incluyeron los 205 pacientes que cumplían los criterios de inclusión señalados previamente. Se excluyeron todos aquellos pacientes usuarios de insulina con otros tipos de diabetes (tipo 2, monogénicas o secundarias a enfermedad pancreática).

Las características demográficas y de la diabetes mellitus se muestran en la Tabla 1.

Sobre el control metabólico, se observó nivel discretamente mayor de HbAlc en menores de 20 años, para volver a elevarse sobre los 50 años. El nivel de HbAlc según grupo etáreo y su desviación estándar se muestran en la Figura 1.

$\mathrm{Al}$ analizar las HbA1c según metas de cumplimiento acorde a criterios de consenso internacional ${ }^{2}, 90$ pacientes $(44 \%)$ presentaban un valor menor o igual a $7,5 \%$, y 59 de ellos $(29 \%)$ presentaban un valor menor o igual a 7,0\%.

\section{Tabla 1. Características demográficas de la muestra de pacientes y datos clínicos sobre la diabetes mellitus}

\begin{tabular}{|lc|}
\hline Variable & Valor \\
\hline Edad (años) & $33 \pm 13$ \\
\hline Sexo femenino/masculino (\%) & $55 / 45$ \\
\hline Tiempo evolución DM1 (años) & $12 \pm 10$ \\
\hline Serología (+) DM1 (\%) & 12 \\
\hline Debut con CAD (\%) & 16 \\
Antecedente familiar DM1 (\%) & 10 \\
\hline IMC (kg/m²) & $25,0 \pm 3,4$ \\
Última HbA1c (\%) & $8,01 \pm 1,67$ \\
\hline
\end{tabular}

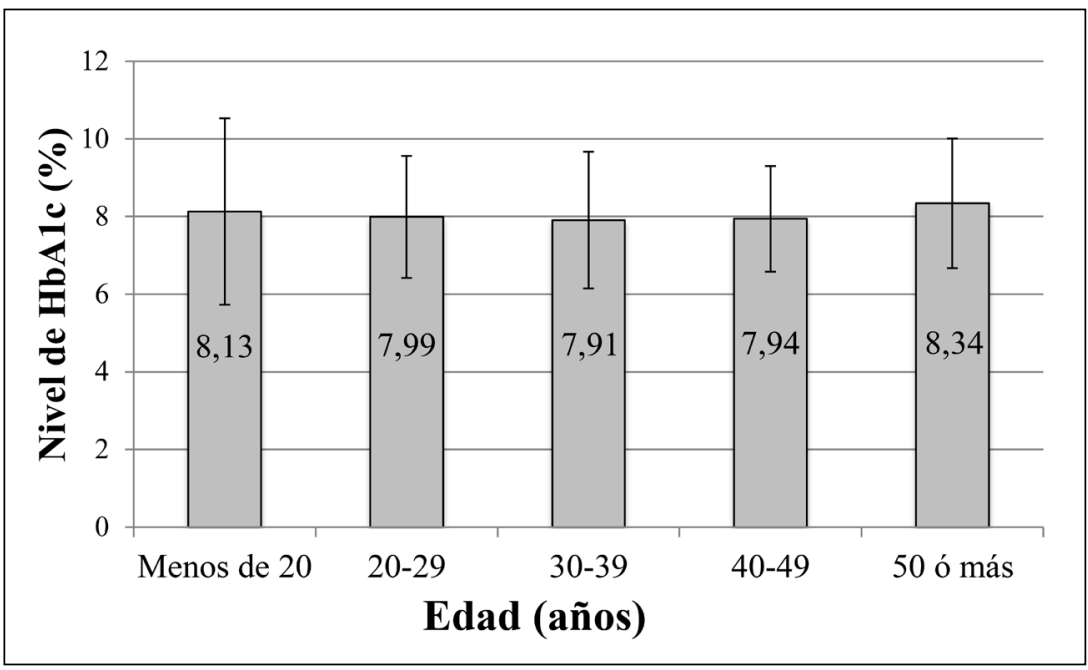

Figura 1. Nivel de $\mathrm{HbA} 1 \mathrm{c}(\%)$ promedio según grupo etáreo. Se muestra el nivel promedio de hemoglobina glicada y su desviación estándar para 5 grupos etáreos distintos. Se observa una tendencia a mayor $\mathrm{HbA} 1 \mathrm{c}$ en menores de 20 y mayores de 50 años. 
La prevalencia de comorbilidades y complicaciones micro y macrovasculares se muestra en la Tabla 2.

Al revisar en detalle los esquemas terapéuticos, $82 \%$ de la muestra analizada se encontraba bajo esquema de múltiples inyecciones diarias (MDI) con lápices o jeringas de insulina, y $18 \%$ con algún tipo de microinfusor contínuo de insulina (CSII). Del total de la muestra analizados, $24 \%$ de los sujetos utiliza en forma rutinaria un monitor flash de glucosa.

De los pacientes con esquema MDI, 35\% de ellos utiliza como insulina basal un análogo lento (glargina-U100 o detemir) y $64 \%$ un análogo ultralento (glargina-U300 o degludec) y $1 \%$ insulina de acción intermedia (NPH).

Como insulina rápida, ya sea en bolos para esquema MDI o como insulina única en pacientes con CSII, 34\% utilizan insulina lispro, 39\% aspart y $24 \%$ glulisina, y en $3 \%$ de los pacientes no se contaba con esta información consignada en su registro. Como método de dosificación de insulina en bolo, ningún paciente lo realiza en dosis fija, 22\% de ellos dosifica con una tabla acorde a glicemia capilar (basada solo en valor de esta, y asumiendo una ingesta de carbohidratos fija) y $78 \%$ con esquema variable (según conteo de carbohidratos a ingerir y factor de corrección según meta y valor de glicemia capilar).

El testeo de la muestra indicó una distribución

\section{Tabla 2. Prevalencia, expresada en porcentaje, de comorbilidades autoinmunes y metabólicas y de complicaciones crónicas, tanto micro como macrovasculares, en la muestra}

\begin{tabular}{|lc|}
\hline Variable & Valor \\
\hline Hipotiroidismo (\%) & 26 \\
\hline Enfermedad celíaca (\%) & 1,4 \\
\hline Insuficiencia suprarrenal (\%) & 0 \\
\hline Otra autoinmune (\%) & 6 \\
\hline Hipertensión arterial (\%) & 12 \\
\hline Dislipidemia & 20 \\
\hline Retinopatía diabética (\%) & 13 \\
\hline Nefropatía diabética (\%) & 2,4 \\
\hline Neuropatía diabética (\%) & 8 \\
\hline Enfermedad cardiovascular (\%) & 2 \\
\hline
\end{tabular}

normal, por lo que se compararon medias mediante test $t$ de Student o test ANOVA. Al comparar las variables predefinidas con el promedio de $\mathrm{HbAlc}$, resultaron significativamente asociados a mejor control la dosificación de insulina rápida mediante esquema variable versus tabla acorde a glicemia capilar (HbA1c 7,85\% versus 8,59\%, $\mathrm{p}=0,008$ ); el uso de CSII versus MDI (HbAlc 7,36\% versus $8,16 \%, \mathrm{p}=0,008)$; $\mathrm{y}$ una dosis de insulina basal menor a $0,4 \mathrm{U} / \mathrm{kg}$ versus una mayor a esa cantidad (HbAlc 7,81\% versus 8,58\%, $\mathrm{p}=0,003$ ). No se encontró diferencias estadísticas en términos de HbAlc al comparar insulina basal ultralenta versus. lenta (HbAlc 8,17 versus $8,16, p=0,986)$, tipo de insulina rápida usada lispro, aspart o glulisina (HbAlc 7,90 versus 7,92 versus 8,4, $\mathrm{p}=0,204$ ), o el uso de MGF versus mediciones de glucosa capilar (HbAlc 8,06 versus 8,00, $\mathrm{p}=0,826$ ). Al comparar el porcentaje de pacientes con $\mathrm{HbAlc}$ menor a 7,5\%, los resultados fueron similares: uso de esquema variable versus tabla acorde a glicemia mostró tendencia pero sin significancia estadística $(47,9 \%$ versus $31,9 \%, p=0,06)$, y fueron significativos el uso de CSSI versus MDI (64,9\% versus $39,8 \%, \mathrm{p}=0,0056)$ y el uso de dosis de insulina basal menor a $0,4 \mathrm{U} / \mathrm{kg}$ versus dosis mayor $(50 \%$ versus $29,1 \%, \mathrm{p}=0,0079$ ).

Los principales resultados que fueron significativamente asociados a mejor control se muestran en las Tablas 3 y 4.

\section{Discusión}

En este estudio se presentan las características demográficas, clínicas, y de esquema de tratamiento de una cohorte de adultos portadores de DM1 atendidos en un centro terciario por un equipo de subespecialistas. Algunas características de esta cohorte son comparables a lo descrito en la literatura, como lo son el nivel de control promedio de la muestra (HbA1c de 8\%), el IMC promedio de la muestra $\left(25 \mathrm{~kg} / \mathrm{m}^{2}\right)$ y la coexistencia de hipotirodismo de $26 \%$, cifras muy similares a las del mismo grupo etáreo en el registro T1D Exchange Clinic Registry ${ }^{5}$, el más grande a nivel mundial, señalado en la introducción de este manuscrito.

Otras cifras encontradas son llamativas, como la prevalencia de enfermedad celíaca bastante más baja a la descrita en distintos registros y series $(1,4 \%$ versus $5 \%)$, lo cual se puede deber 
Tabla 3. Valor promedio de HbA1c entre distintas comparaciones, diferencia absoluta entre los grupos y magnitud de asociación estadística

\begin{tabular}{|c|c|c|c|}
\hline Comparación & A1c (\%) & Diferencia absoluta (\%) & $\mathbf{p}$ \\
\hline $\begin{array}{l}\text { Forma de dosificar insulina rápida: } \\
\text { Esquema variable } \\
\text { vs } \\
\text { Tabla fija }\end{array}$ & $\begin{array}{l}7,85 \\
8,59\end{array}$ & $-0,74$ & 0,008 \\
\hline $\begin{array}{l}\text { Método de administración de insulina: } \\
\text { Bomba de insulina } \\
\text { vs } \\
\text { Múltiples inyecciones }\end{array}$ & $\begin{array}{l}7,36 \\
8,16\end{array}$ & $-0,80$ & 0,008 \\
\hline $\begin{array}{l}\text { Dosis de insulina basal } \\
<0,4 \mathrm{U} / \mathrm{kg} \\
\text { vs } \\
>0,4 \mathrm{U} / \mathrm{kg}\end{array}$ & $\begin{array}{l}7,85 \\
8,58\end{array}$ & $-0,73$ & 0,003 \\
\hline
\end{tabular}

Tabla 4. Porcentaje de pacientes que consiguen una A1c menor o igual a 7,5\% (considerada como meta de "buen control metabólico") entre distintas comparaciones, diferencia absoluta entre los grupos y magnitud de asociación estadística

\begin{tabular}{|c|c|c|c|}
\hline Comparación & $\begin{array}{c}\text { Porcentaje de pacientes } \\
\text { con A1c }<7,5 \%(\%)\end{array}$ & $\begin{array}{c}\text { Diferencia } \\
\text { absoluta (\%) }\end{array}$ & $\mathbf{p}$ \\
\hline $\begin{array}{l}\text { Forma de dosificar insulina rápida: } \\
\text { Esquema variable } \\
\text { vs } \\
\text { Tabla fija }\end{array}$ & $\begin{array}{l}47,9 \\
31,9\end{array}$ & +16 & 0,06 \\
\hline $\begin{array}{l}\text { Método de administración de insulina: } \\
\text { Bomba de insulina } \\
\text { vs } \\
\text { Múltiples inyecciones }\end{array}$ & $\begin{array}{l}64,9 \\
39,8\end{array}$ & $+25,1$ & 0,006 \\
\hline $\begin{array}{l}\text { Dosis de insulina basal } \\
<0,4 \mathrm{U} / \mathrm{kg} \\
\quad \text { vs } \\
>0,4 \mathrm{U} / \mathrm{kg}\end{array}$ & $\begin{array}{r}50 \\
29,1\end{array}$ & $+20,9$ & 0,008 \\
\hline
\end{tabular}

a un subdiagnóstico de esta enfermedad, lo cual debe ser explorado con mayor detalle a futuro en nuestra cohorte. Es llamativa, además, la prevalencia relativamente baja de complicaciones microvasculares; si bien nos gustaría atribuir esta cifra al hecho de que estos pacientes se controlan en un centro terciario altamente especializado en el área, probablemente la explicación más correcta sea que se trata de una población con DM1 de moderado tiempo de evolución (en promedio 12 años), que aún no ha tenido tiempo de desarrollar complicaciones. Otra característica relevante de la muestra es la alta tasa de uso de los análogos de acción ultralenta como insulina basal (glar-
gina-U300 o degludec), la cual se explica porque nuestra red ambulatoria es prestador de la canasta de garantías explícitas de salud (GES) de DM1, de dos aseguradoras que cubren estas insulinas en todo paciente con DM1.

Una deficiencia importante de nuestro análisis es que se trata de un estudio retrospectivo con comparación de valores promedios de HbAlc y sin un método sistemático para determinar la prevalencia y gravedad de la hipoglucemia. Lo anterior podría explicar que el tipo de insulina basal y rápida usada, y el uso de MGF, no hayan resultado significativamente asociadas a mejor $\mathrm{HbAlc}$, pues estas tres intervenciones terapéuticas 
han mostrado en forma consistente mantener el control metabólico (medido como HbAlc) con reducción de la tasa de hipoglucemia en diferentes escenarios clínicos $^{9-11}$. Este análisis es clave, pues como clínicos debemos seguir estimulando el uso de estas y otras intervenciones que reduzcan las hipoglucemias, dado que esto, sumado a la mantención o mejoría del promedio glicémico (HbAlc), aumenta el tiempo en rango deseado de glicemias, un objetivo altamente deseable en el control de pacientes con DM1 en la actualidad, tanto por mejorar calidad de vida, como por el riesgo que conlleva la hipoglucemia. Otra limitación de nuestro estudio es que las HbAlc no fueron todas tomadas en el mismo laboratorio, aunque un gran porcentaje de ellas fueron realizadas en el laboratorio de la Red Salud UC.

Es importante analizar con mayor detalle las variables que sí resultaron significativamente asociadas. Al igual que el estudio $\mathrm{DAFNE}^{6}$, en nuestro estudio, el grupo que utilizaba un esquema variable de insulina rápida, que considera que el paciente ajuste sus dosis de insulina en relación a la cantidad de carbohidratos que va a comer y la glicemia capilar, reportó mejores niveles de $\mathrm{HbAlc}$, en comparación con los que usaban tabla acorde exclusivamente a glicemia capilar. Asimismo, el uso de microinfusores de insulina, que requiere de una gran intervención educativa, incluido el conteo de carbohidratos para la dosificación variable de insulina rápida, y un paciente mucho más protagónico en su autocontrol, sumado al mejor método de infusión más fisiológica en microdosis en el subcutáneo, permite explicar este mejor control. Finalmente, el uso de una basal definida en muchas publicaciones como fisiológica, es decir similar a la cantidad de insulina de secreción estable en las personas sin diabetes, numéricamente $<0,4 / \mathrm{u} / \mathrm{kg}$ por día, se asocia a un uso adecuado de la insulina rápida con cada ingesta de carbohidratos, que requiere, a su vez, importante educación en conteo de carbohidratos y autocontrol, limitando grandes excursiones glicémicas, explicando así este notable mejor control.

Del análisis de esta cohorte podemos concluir que la clave del éxito en la terapia de la DM1 está asociada especialmente a intervenciones educativas que permitan al paciente una mejor comprensión y autocontrol de su enfermedad en los distintos momentos y situaciones de cada día. No se debe olvidar que, si bien este análisis es incapaz de pesquisarlo, la hipoglucemia es un evento indeseable que forma parte de un buen manejo metabólico de la DM1 y que debe ser prevenido y evitado dentro de lo posible, mediante la educación en el correcto uso de la insulina y su administración en las diferentes situaciones del día a día y en la educación en conteo de carbohidratos.

\section{Referencias}

1. Chiang JL, Kirkman MS, Laffel LM, Peters AL. Type 1 Diabetes Through the Life Span: A Position Statement of the American Diabetes Association. Diabetes Care 2014; 37 (7): 2034-54.

2. American Diabetes Association. Pharmacologic Approaches to Glycemic Treatment: Standards of Medical Care in Diabetes-2018. Diabetes Care 2018; 41 (Supplement 1): S73-S85.

3. Nathan DM, Genuth S, Lachin J, Cleary P, Crofford O, Davis M, et al; Diabetes Control and Complications Trial Research Group. The effect of intensive treatment of diabetes on the development and progression of long-term complications in insulin-dependent diabetes mellitus. N Engl J Med 1993; 30; 329 (14): 977-86.

4. The Diabetes Control and Complications Trial (DCCT)/ Epidemiology of Diabetes Interventions and Complications (EDIC) Study Research Group. Mortality in Type 1 Diabetes in the DCCT/EDIC Versus the General Population. Diabetes Care 2016; 39 (8): 1378-83.

5. Miller KM, Foster NC, Beck RW, Bergenstal RM, DuBose SN, DiMeglio LA, et al. Current State of Type 1 Diabetes Treatment in the U.S.: Updated Data From the T1D Exchange Clinic Registry. Diabetes Care 2015; 38: 971-8.

6. Mansell P. The Dose Adjustment for Normal Eating (DAFNE) education programme. J Diabetes Nurs 2012; 16 (9): 364-9.

7. Doyle EA, Weinzimer SA, Steffen AT, Ahern JH, Vincent M, Tamborlane WV. A Randomized, Prospective Trial Comparing the Efficacy of Continuous Subcutaneous Insulin Infusion With Multiple Daily Injections Using Insulin Glargine. Diabetes Care 2004; 27: 1554-8.

8. Bergenstal RM, Tamborlane WV, Ahmann A, Buse JB, Dailey G, Davis SN, et al. Effectiveness of Sensor-Augmented Insulin-Pump Therapy in Type 1 Diabetes. N Engl J Med 2010; 363: 311-20.

9. Mathieu C, Hollander P, Miranda-Palma B, Cooper J, Franek E, Russell-Jones D, et al. Efficacy and safety of insulin degludec in a flexible dosing regimen versus 
insulin glargine in patients with type 1 diabetes (BEGIN: Flex T1): a 26-week randomized, treat-to-target trial with a 26-week extension. J Clin Endocrinol Metab 2013; 98 (3): 1154-62.

10. Home PD, Bergenstal RM, Bolli GB, Ziemen M, Rojeski M, Espinasse M, et al. New Insulin Glargine 300 Units/ $\mathrm{mL}$ Versus Glargine 100 Units/mL in People With Type 1 Diabetes: A Randomized, Phase 3a, Open-Label Clinical Trial (EDITION 4). Diabetes Care 2015; 38 (12): 2217-25.

11. Bolinder J, Antuana R, Geelhoed-Duijvestin P, Kröger J, Weitgasser R. Novel glucose-sensing technology and hypoglycaemia in type 1 diabetes: a multicentre, nonmasked, randomised controlled trial. Lancet 2016; 388 (10057): 2254-63.

12. Carrasco E, Angel B, Codner E, García D, Ugarte F, Bruzzone ME, Pérez F. Incidencia de diabetes mellitus tipo 1 en Santiago de Chile: análisis por comunas de la Región Metropolitana en el período 2000-2004. Rev Med Chile 2006; 134 (10): 1258-64.

13. Hodgson MI, Ossa JC, Velasco N, Urrejola P, Arteaga A. Cuadro clínico de inicio de la diabetes tipo 1 en el niño. Rev Med Chile 2006; 134: 1535-40.

14. Ferreira-Hermosillo A, Molina-Ayala MA. Enfermedades autoinmunitarias asociadas a diabetes mellitus tipo 1A. Rev Med Chile 2015; 143 (8): 1042-9.

15. Díaz-Cárdenas C, Wong C, Vargas Catalán N. Grado de control metabólico en niños y adolescentes con diabetes mellitus tipo 1. Rev Chil Pediatr 2016; 87(1): 43-7.

16. Codner Ethel, Mericq V, García H, López C, Cáceres J, Gaete X, Avila A. Resultados de un programa multidisciplinario de tratamiento intensificado de la Diabetes Mellitus tipo 1 (DM1) en un Hospital Público. Rev Med Chile 2001; 131 (8): 857-64. 